\section{Ethanol-Locks schützen Kinder vor ZVK-Infektion}

Die Prävention von Infektionen der Blutbahn ist gerade bei Kindern mit Krebs, die mit zentralen Venenkathetern versorgt werden, wichtig. Hierfür sind Ethanol-Locks empfehlenswert, wie sich in einer aktuellen Studie zeigte.

E than thanol-Locks sind eine vielversprechende Option zur Prävention katheterassoziierter Infektionen. Ethanol kann die pathogene Besiedelung zentraler Venenkatheter (ZVK) eliminieren und ist leicht anzuwenden, Resistenzen sind selten. Nun wurde erstmals in einer randomisierten, kontrollierten Studie untersucht, ob sie solche Infektionen bei Kindern verhindern können. 307 Krebspatienten im Alter von 1-18 Jahren erhielten in den ersten 4 Wochen nach Neuanlage eines ZVK randomisiert für 2 Stunden entweder Ethanol- (70 \%) oder Heparin-Locks (100 IU/ml). Primäre Endpunkte waren die Zeit bis zu einer katheterbedingten Infektion der Blutbahn oder bis zum Tod aufgrund einer solchen Infektion.
In der Ethanol-Gruppe entwickelten $10 \%$ der Patienten eine solche Infektion, in der Heparin-Gruppe 19\% (Hazard Ratio [HR] 0,53 ). Die Inzidenz betrug 0,77 vs. $1,46 / 1.000$ Katheter-Tage ( $p=0,039$ ). Die ,number needed to treat" $z$ ur Prävention einer Infektion durch Ethanol-Locks war 13. Nach Adjustierung bezüglich ZVK-Typ und Diagnose lag die Hazard Ratio für die Entwicklung einer Infektion der Blutbahn in der Ethanol-Gruppe bei 0,57 im Vergleich zur Heparin-Gruppe. Kein Patient starb an einer katheterbedingten Infektion der Blutbahn. Die meisten Infektionen (64\%) wurden durch grampositive Bakterien verursacht, die Ethanol-Locks besonders stark reduzieren (Ethanol: $\mathrm{n}=8$; Heparin: $\mathrm{n}=21 ; \mathrm{p}=0,012)$. In der Ethanol-Grup- pe mussten weniger ZVK aufgrund einer katheterbedingten Infektion entfernt werden ( 3 vs. $8 \%$; $\mathrm{p}=0,077$ ).

Patienten mit Ethanol-Locks hatten signifikant mehr vorübergehende unerwünschte Symptome wie Nausea, Veränderungen des Geschmackssinns und Benommenheit, wobei alle Symptome höchstens Grad 2 erreichten und vorübergehend waren. Hepatotoxizitäten und ein Anstieg der Leberwerte traten in beiden Gruppen vergleichbar häufig auf. Es wurden keine Verdachtsfälle unerwarteter, schwerwiegender, unerwünschter Ereignisse beobachtet.

Fazit: Dies ist die erste randomisierte Studie, in der sich zeigte, dass Ethanol-Locks krebskranke Kinder vor ZVK-assoziierten Blutbahninfektionen schützen. Die Forscher empfehlen ihre Anwendung in der klinischen Praxis. Judith Neumaier

Schoot RA et al. Prevention of central venous catheter-associated bloodstream infections in paediatric oncology patients using $70 \%$ ethanol locks: A randomised controlled multi-centre trial. Eur J Cancer. 2015;51(14):2031-8.

\title{
Hohes Sturzrisiko bei älteren Krebspatienten
}

\begin{abstract}
Im höheren Lebensalter zu stürzen birgt ein hohes Risiko für anhaltende Bewegungseinschränkungen, Hospitalisierung und den Verlust an Selbstständigkeit. Wie verhält es sich bei älteren Patienten mit malignen Erkrankungen?
\end{abstract}

S chätzungen zufolge stürzt nahezu ein $\int$ Drittel aller selbstständig lebenden älteren Menschen jenseits des 65. Lebensjahres mindestens 1-mal pro Jahr. Die Gründe für die hohe Inzidenz sind vielschichtig: chronische Erkrankungen, allgemeine Muskelschwäche, Medikamente, Einschränkungen des Sehvermögens ebenso wie schlechtes Schuhwerk oder Fallen im Haushalt. Ältere Menschen mit einer malignen Erkrankung sind offenbar verstärkt sturzgefährdet.

Für die Studie wurden 1.172 Patienten im Durchschnittsalter von 73 Jahren gewonnen, davon $74 \%$ Frauen. Alle lebten selbstständig. Die Mehrheit litt an Brustkrebs (55\%) oder einer hämatologischen Neoplasie (14\%). Mithilfe des „comprehensive geriatric assessment" (CGA) wurden die Patienten zu verschiedenen
Zeitpunkten vor, während und nach der Therapie befragt.

256 (22\%) Patienten berichteten, innerhalb der letzten 6 Monate gestürzt zu sein: 140 (12\%) 1-mal, 66 (6\%) 2-mal, 16 (1\%) dreimal und $34(3 \%) \geq 4$-mal.

Patienten, die mindestens einen Sturz erlebt hatten, waren etwas älter $(p=0,03)$. Geschlecht, Herkunft, Schulbildung, Ehestatus, Krebsart oder Therapiephasen spielten keine Rolle. Patienten, die für die Verrichtung normaler Alltagstätigkeiten mindestens ein technisches Hilfsgerät in Anspruch nahmen oder körperliche Einschränkungen hatten, waren gefährdeter als Patienten ohne Handicap $(p \leq 0,001)$. Auch die Einnahme von Medikamenten, Begleiterkrankungen, eine Zeit von mehr als 14 Sekunden im Aufsteh- und Gehtest und ein eingeschränktes Sehvermögen waren mit einem signifikant höheren Sturzrisiko verbunden $(p \leq 0,001)$. Eingeschränkte physische Funktionen, schlechtes Sehvermögen und niedriger Performancestatus hatten die höchsten Odds Ratios $(3,6 ; 3,4 ; 3,0)$ für Stürze. Von Begleiterkrankungen waren vor allem Arthritis, Kreislaufprobleme, Insult und Depression mit einem signifikant erhöhten Sturzrisiko assoziiert, nicht hingegen Hypertonie, Diabetes mellitus oder Herzerkrankungen. Mit jeder zusätzlichen Komorbidität stieg die Odds Ratio für Stürze um $20 \%$.

Fazit: Ältere Krebspatienten, die selbstständig leben, haben ein hohes Sturzrisiko. Komorbiditäten, eingeschränkte körperliche Funktionen, Medikation, Sehstörungen und Benutzung technischer Hilfen im Alltag waren signifikant damit assoziiert. Kathrin von Kieseritzky

Williams G. R. et al. Geriatric assessment as an aide to understanding falls in older adults with cancer. Support Care Cancer. 2015;23(8):2273-80. 